\title{
An investigation of ethical conflict, ethical competency and educational needs for the organ transplant coordinators
}

\author{
Sunyoung Son ${ }^{1}$, Jayoung You ${ }^{2}$, Ilhak Lee ${ }^{3}$, Myoung Soo Kim ${ }^{4}$ \\ ${ }^{1}$ Department of Surgery-Transplantation, Gangnam Severance Hospital, Seoul, Korea
${ }^{2}$ Human Research Protection Center, Severance Hospital, Seoul, Korea
${ }^{3}$ Graduate School of Public Health, Yonsei University, Seoul, Korea
${ }^{4}$ Department of Surgery-Transplantation, Severance Hospital, Seoul, Korea
}

Background: It is a descriptive research study attempted to revitalize ethical education to reduce ethical conflicts and improve ethical competencies by identifying the ethical conflict, competency, job satisfaction, turnover intention, and ethical education needs of organ transplant coordinators in clinical practice.

Methods: Survey data were collected from 87 people who agreed to the online survey from March 22 to May 28, 2021, with about 160 people registered with the Korea Organ Transplant Coordinator Association working as organ transplant coordinators at 107 hospitals in Korea. For ethical conflict and decision-making measurement, the tool developed by Son Hee-jin (2000), the tool used by Park Jung-hye (2002), the tool used by Kim Mi-ran (2007), the ethical competency tool was revised and supplemented, and the tool developed to find out the need for education. The collected data were analyzed using SAS using independent t-test, ANOVA, Pearson's correlation, and multiple regression analysis.

Results: In general characteristics, it was $87.4 \%$ for women and the average age was 38.9 years old $( \pm 6.72)$. The average nurse experience was 172.2 months $( \pm 89.50)$ and the organ transplant coordinator experience was 89.3 months $( \pm 59.89)$. The tasks in charge were brain-dead only $46.0 \%$, transplanted only $28.7 \%$, and brain-dead+transplanted $25.3 \%$. Ethical conflict experience averaged 3.5 points $( \pm 0.86)$, the lower area of ethical conflict averaged 3.8 points $( \pm 1.01)$, the average conflict with the medical team averaged 3.7 points $( \pm 1.02)$, and the average conflict with brain-dead care averaged 2.5 points $( \pm 0.96)$. In terms of the degree of consideration when making ethical decisions, the doctor's order was 4.9 points $( \pm 0.82)$, lower than other items, but when asked which opinions were reflected in the decision-making when ethical problems occurred, the doctor was the highest at $62.1 \%$. The average degree of ethical competency was 4.7 points $( \pm 0.51)$, followed by an average willingness to ethics 5.2 points $( \pm 0.61)$, an average technology for ethical practice 4.7 points $( \pm 0.53)$, an average self-awareness 4.7 points $( \pm 0.61)$, and an average strength 4.3 points $( \pm 0.70)$. As for the ethics education needs, the average score for ethics education needs was 5.0 points $( \pm 0.94)$, and ethics related to organ transplantation was the highest at 73.6\%. Ethical conflict according to general characteristics differed significantly depending on the task in charge of transplant patients and their family relations $(t=3.03, P=0.003)$, and it was confirmed that there was a significant difference according to the experience of organ transplant coordinator in the area of professional work $(t=3.32, P=0.001)$. Ethical competencies according to general characteristics were found to have significant differences according to the experience of organ transplant coordinator in the field of awareness $(t=-2.21, P=0.030)$. Ethical conflict is about turnover intention $(r=0.472, P<0.05)$, job satisfaction is ethical competency $(r=0.277, P<0.05)$. Regarding the need for ethics education $(r=0.229, P<0.05)$, ethical competency showed a significant positive correlation with the need for ethics education $(r=0.474$, $P<0.05)$. As the turnover intention increased, the demand for ethics education increased significantly $(t=2.46, P=0.016)$, and as the ethical competency increased, the demand for ethics education increased significantly $(t=4.94, P<0.001)$.

Conclusions: A significant difference in the degree of ethical conflict according to general characteristics was confirmed, and organ transplant coordinators were a group with high ethical and ethical education needs, and the higher the ethical competency, the higher the ethical education demand. Based on the research results, ethics education suitable for organ transplant coordinators should be developed, standardized ethics education for supporting ethical decisions, establishing moral ethics of professionals, and ethics education applicable to clinical sites.

Corresponding author: Jayoung You

E-mail: prettywoman725@yuhs.ac 